\title{
The RBO dataset of articulated objects and interactions
}

The International Journal of Robotics Research

2019, Vol. 38(9) 1013-1019

(C) The Author(s) 2019

Article reuse guidelines:

sagepub.com/journals-permissions DOI: $10.1177 / 0278364919844314$

journals.sagepub.com/home/ijr

\author{
Roberto Martín-Martín* (D), Clemens Eppner* and Oliver Brock
}

\begin{abstract}
We present a dataset with models of 14 articulated objects commonly found in human environments and with RGB-D video sequences and wrenches recorded of human interactions with them. The 358 interaction sequences total 67 minutes of human manipulation under varying experimental conditions (type of interaction, lighting, perspective, and background). Each interaction with an object is annotated with the ground-truth poses of its rigid parts and the kinematic state obtained by a motion-capture system. For a subset of 78 sequences (25 minutes), we also measured the interaction wrenches. The object models contain textured three-dimensional triangle meshes of each link and their motion constraints. We provide Python scripts to download and visualize the data. The data are available at https://turbo.github.io/articu lated-objects/ and hosted at https://zenodo.org/record/1036660/.
\end{abstract}

\section{Keywords}

Articulated objects, manipulation, interaction, real-world data

\section{Introduction}

The RBO dataset is a collection of 358 RGB-D video sequences (67 minutes) of humans manipulating 14 articulated objects under varying experimental conditions (type of interaction, lighting, perspective, and background). All sequences are annotated with ground truth of the poses of the rigid parts and the kinematic state of the articulated object (joint states) obtained with a motion-capture system. We also provide kinematic models of these objects including 3D textured shape models. For 78 sequences ( 25 minutes) the interaction wrenches during the manipulation are also recorded.

We present the first dataset with articulated objects. All similar datasets contain single rigid-body objects that move or are being manipulated. There are two datasets that could be considered close to ours. The first (Garcia Cifuentes et al., 2017) is a dataset that was released together with a method for robot arm tracking. This dataset contains images and joint encoder values of a moving robot arm and its kinematic and shape model. In contrast, our dataset is targeted to the study of interactions with everyday human objects: it contains models of multiple ubiquitous articulated objects and sequences of interactions in varying environmental conditions. The second dataset (Michel et al., 2015) provides models of four everyday articulated objects and ground truth of the static pose of each link and the changing pose of camera during the video sequence. The sequences of this dataset do not contain any interaction or manipulation of the objects, only camera motion. Therefore, neither of these datasets can be used to study interactions and to evaluate methods for perceiving them.

Our dataset will help evaluate algorithms for tracking articulated objects (e.g. Schmidt et al., 2014) and building models of articulated objects (e.g. Martín-Martín et al., 2016). Apart from benchmarking, the dataset can also be used to develop data-driven algorithms by exploiting the provided models to generating virtual visual data. Although there is a vast amount of 3D models and datasets of objects (Calli et al., 2015; Kasper et al., 2012), very few of them include and describe articulated mechanisms.

\section{Sensor setup}

We use the following sensors to record human interactions with articulated objects (see Figure 1).

- RGB-D camera Asus Xtion Pro Live, $640 \times 480$ pixels, $30 \mathrm{~Hz}$, pointed at the object.

Robotics and Biology Laboratory, Technische Universität Berlin, Germany

*These authors contributed equally.

Corresponding author:

Roberto Martín-Martín, Robotics and Biology Laboratory, Marchstr. 23, 10587 Berlin, Germany.

Email: roberto.martinmartin@tu-berlin.de 


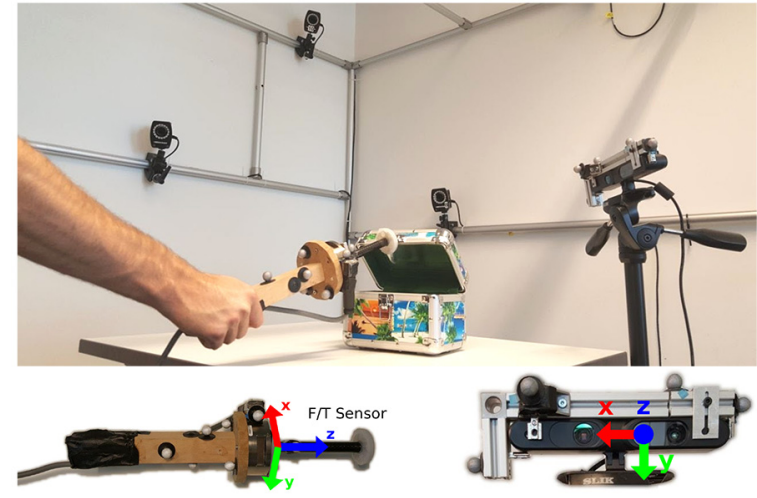

Fig. 1. Our sensor setup for recording interactions with articulated objects. Top: interaction in the motion capture volume. Bottom left: tool with force/torque sensor for recording interaction wrenches, motion capture markers, and measurements reference frame. Bottom right: Asus RGB-D sensor with motioncapture markers and measurements reference frame.

- Motion-capture system by Motion Analysis (2018) (Cortex SDK 1.10 running on Windows 10), capture volume: SI $1.5 \mathrm{~m}^{3}$ including 18 Osprey cameras, providing 3D positions of fiducial markers at $100 \mathrm{~Hz}$.

- Force/torque (F/T) sensor ATI FTN-Gamma DAQ/Net, calibration SI-130-10, force/torque resolution: $F_{x}=F_{y}=\mathrm{SI}[$ quotient - mode $=$ fraction $] 1 / 40 \mathrm{~N}$, $F_{z}=\mathrm{SI}[$ quotient - mode $=$ fraction $] 1 / 20 \mathrm{~N}$, $T_{x}=T_{y}=T_{z}=\mathrm{SI}[$ quotient - mode $=$ fraction $] 1 / 800$ $\mathrm{Nm}$, recorded at $100 \mathrm{~Hz}$.

The three sensor modalities are recorded on a single machine using Ubuntu 16.04 and ROS Indigo. We neglect time delays imposed by different data transport mechanisms (images are streamed via USB, whereas $\mathrm{F} / \mathrm{T}$ and motion capture data are streamed via Ethernet on TCP/IP protocol). Upon data inspection we did not observe synchrony issues.

We acquired separately 3D triangle meshes of each articulated object part, using the following sensors and methods.

- Structured light scanning system by Hewlett-Packard (2018), SLS-3 HD, scan size 60-500 mm, resolution down to $0.05 \mathrm{~mm}$.

- Reconstruction software Autodesk (2018), used with photos taken with a Casio Exilim EX-FC100, 9 MP.

\section{Data structure and usage}

The RBO dataset is available at https://tu-rbo.github.io/ articulated-objects/ and hosted at https://zenodo.org/record/ 1036660/. It is composed of two parts (see Figure 2): a first part with descriptions of 14 articulated objects and the main part containing 358 human interactions with these objects. We also provide Python scripts to facilitate downloading, visualizing and working with the data.

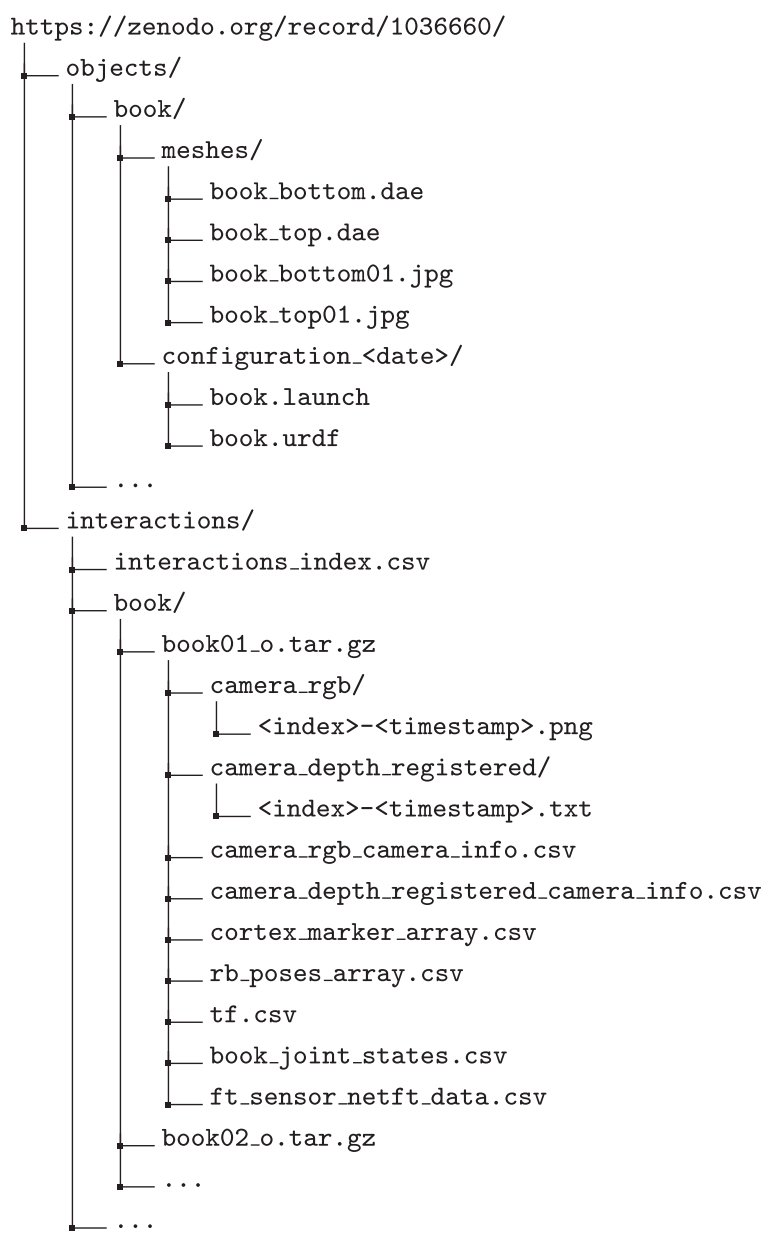

Fig. 2. The dataset is structured by objects and interactions. Please see the text for details.

\subsection{Models of articulated objects (objects /)}

The RBO dataset contains 14 models of articulated mechanisms that are commonly found in human environments. Table 1 describes these objects and their kinematic structure. Each object model (<object_id $>$ /) consists of the following.

- Link geometries (meshes/): We describe the shape of a link as a 3D triangle textured mesh in the COLLADA (2012) format (<part_name>.dae). We provide the associated texture as JPEG images (<part name $><$ index $>$.jpg).

- Kinematic structure (configuration_<date $>/$ ): We define the relation of links and joints with the widely used Unified Robot Description Format (URDF, 2018) (<object_id>.urdf), an XML file format to describe all elements of articulated objects with a chain or tree structure. The objects of the database possess one-degree-of-freedom (one-DoF) joints that can be either prismatic or revolute joints. A prismatic joint constrains the relative motion between a pair of bodies to a pure translation along the prismatic axis, while a 
Table 1. The dataset contains 14 articulated objects. Letters in the last column represent (S)tatic, (F)loating, (R)evolute, and (P)rismatic joints.

\begin{tabular}{|c|c|c|}
\hline Object ID & Picture & Actuated joints \\
\hline globe & & $\mathrm{F}-\mathrm{R}$ \\
\hline laptop & & $\mathrm{F}-\mathrm{R}$ \\
\hline ikea & & $-\mathrm{R}$ \\
\hline foldingrule & & $\mathrm{F}-\mathrm{R}-\mathrm{R}$ \\
\hline book & & $\mathrm{F} \longrightarrow \mathrm{R}$ \\
\hline treasurebox & & $\mathrm{F}-\mathrm{R}$ \\
\hline tripod & & $\mathrm{F}-\mathrm{P}-\mathrm{R}$ \\
\hline clamp & & $\mathrm{F} \longrightarrow \mathrm{P}$ \\
\hline pliers & & $\mathrm{F}-\mathrm{R}$ \\
\hline cardboardbox & & $\mathrm{F}-\mathrm{R}$ \\
\hline
\end{tabular}

Table 1. Continued

\begin{tabular}{|c|c|c|}
\hline Object ID & Picture & Actuated joints \\
\hline rubikscube & & $\mathrm{F} \longrightarrow \mathrm{R}$ \\
\hline microwave & & $\mathrm{S}-\mathrm{R}$ \\
\hline ikeasmall & & \\
\hline cabinet & & \\
\hline
\end{tabular}

revolute joint constraints the motion to a pure rotation around the rotational axis. The base link is the origin of the kinematic tree or chain. It is either rigidly connected to the environment (represented with a static joint with zero DoFs) or completely unconstrained (represented with a floating joint with six DoFs). The reference coordinate frame of a link corresponds to a marker set of the motion-capture system (see Section 4). We define the joint parameters and link meshes with respect to these coordinate frames. As marker set locations can vary between recording sessions, we provide a separate kinematic structure description for each session indicated by the $<$ date $>$ suffix in the folder name.

\subsection{Interactions (interactions/)}

The RBO dataset contains sensor data of 358 human interactions with the 14 modeled objects ( $\geqslant 25$ interactions per object). The sequences last between 2.7 and $69.0 \mathrm{~s}$ (median: $9.15 \mathrm{~s}$ ). They differ in lighting conditions, camera perspective and motion, background, clutter, actuation of the mechanisms, and human motion (see Table 2). The file interactions index. CSV contains a list of all interactions and their properties.

The sensor data are organized per object (<object $i d>/$ ) and interaction (<object_id $\left.><i n d e x>\_o /\right)$. Each interaction includes the following: 
Table 2. Properties of the 358 interaction recordings.

\begin{tabular}{lll}
\hline Lighting Conditions & Artificial & 178 \\
& Natural & 107 \\
& Dark & 73 \\
Camera Motion & Yes & 100 \\
& No & 258 \\
Type of Background & Plain & 197 \\
& Textured & 91 \\
& Black & 70 \\
Clutter & Yes & 109 \\
& No & 249 \\
Actuated DoFs & Only internal & 162 \\
& Internal and external & 196 \\
Interaction Wrenches & Yes & 78 \\
& No & 280 \\
\hline
\end{tabular}

- RGB images: We store the color images as 8-bit loss-less compressed PNG files (camera_rgb/ <index >-<timestamp >.png).

- Depth images: We register the depth images to the RGB camera frame (see Section 4) and store them as text files containing distances in meters (camera_depth_registered / $<$ index $>$ - <timestamp $>$ .txt).

- Intrinsic camera parameters: We provide the focal length, center point, and distortion parameters of the Plumb Bob model (Brown, 1966) for both the camera that generates RGB (camera_rgb camera info.CSV) and the camera that generates depth images (camera_depth_registered camera info.csv).

- Extrinsic camera parameters: We represent the 6D transformation between the cameras of the RGB-D sensor with a translation vector and a quaternion (tf.csv).

- Infrared marker positions: We include the 3D locations of all infrared fiducial markers in the scene measured by the motion-capture system (cortex_markers_array.csv).

- Rigid body poses: We store the $6 \mathrm{D}$ poses defined by sets of infrared fiducial markers as position vectors and quaternions. We include the pose of each link of the articulated object, the RGB-D and F/T sensor at 100 $\mathrm{Hz}$ (rb_poses_array.csv).

- Joint configurations: We compute the joint configuration of the articulated object in the scene from the $6 \mathrm{D}$ poses of its links (<object>_joint_states . CSv).

- Wrenches: We provide the forces and torques for the interaction as provided by the F/T sensor ( $\mathrm{ft}$ ssensor_netft_data.csv). We include this data in at least five interactions per object.

Note that we do not provide data about human arm postures. Although the data can be used to analyze human interactions, our focus is on the state of articulated objects during interaction. Human poses could be obtained by applying existing methods on the provided camera images such as that described by Cao et al. (2017).

\subsection{Utilities}

We provide Python scripts and a ROS package on the website https://tu-rbo.github.io/articulated-objects/ to facilitate the download and visualization of the data.

- The download script (rbo_downloader.py) fetches object models and interaction files. The user can also select groups of interactions fulfilling a certain property, e.g. all interactions with an object or all interactions with wrench measurements.

- The visualization script (rbo_visualizer.py) displays the content of an interaction folder: RGB, depth images, wrenches, and/or joint states.

- ROS package: In addition to the interaction sequences in the file format described above, we also provide all data in the form of a rosbag (ROS, 2018). We provide a ROS package including scripts to visualize the data in this format.

We encourage users of the dataset to help extend it by adding new articulated objects. Instructions on how to contribute are available on the dataset's website: https://tu-rbo. github.io/articulated-objects/\#contributing.

\section{Data acquisition}

\subsection{Visual data and $6 D$ body poses}

The main goal of our dataset is to evaluate and develop algorithms based on visual data (RGB or RGB-D) with/ without interaction wrenches for the perception of articulated objects. For this goal, it is crucial to register accurately the visual information and the ground truth provided by the motion-capture system. We first calibrate the intrinsic parameters of the RGB-D sensor. We use a checkerboard of known dimensions and take pictures at different poses of the checkerboard with respect to the camera with both the 

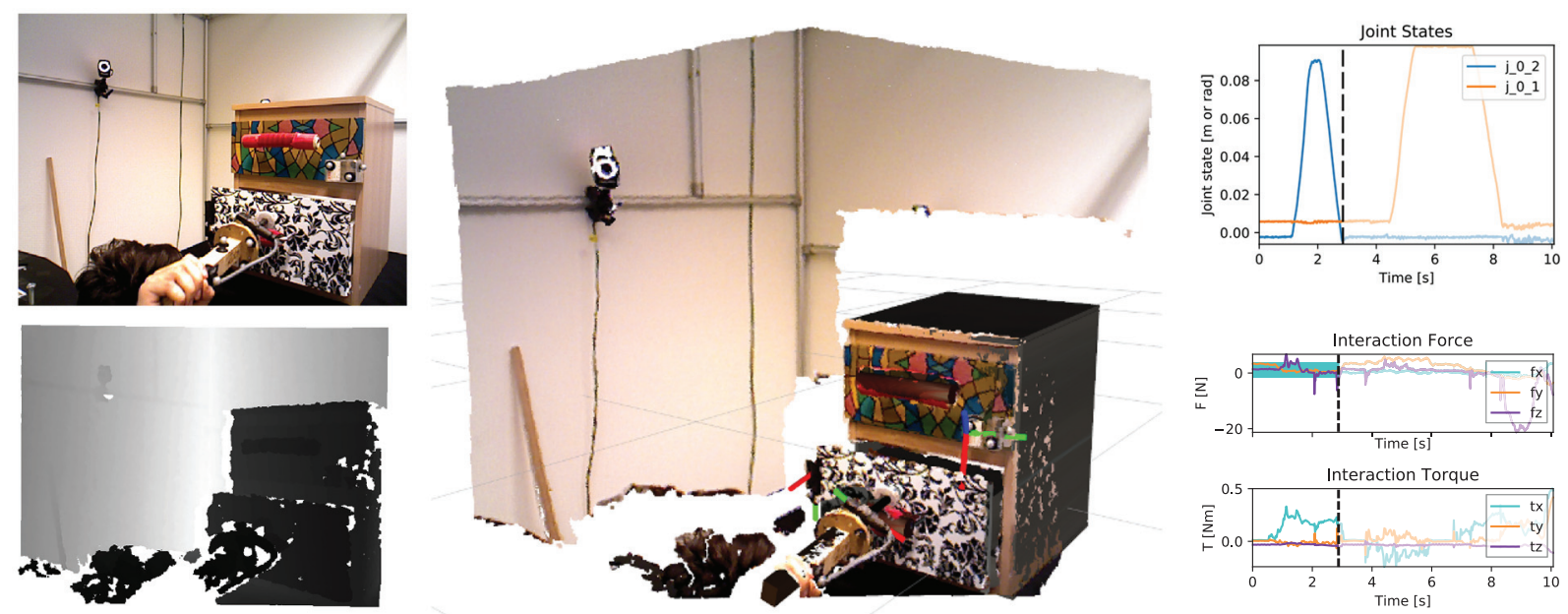

Fig. 3. Visualization of the sensor data for opening a drawer: RGB and depth image (left), mesh model, point cloud, and coordinate frames of tracked bodies (middle), and drawer state and applied wrenches (right) with a dashed vertical line indicating the current time.

RGB and the infrared camera of the RGB-D sensor. We use an OpenCV-based camera calibration tool to estimate the internal parameters of the cameras (focal length, center point, and distortion parameters of the Plumb model) by detecting corner points on the checkerboard and estimating the parameters that minimize the squared error of the reprojection of the points from a separate multi-view perspective- $n$-point $(\mathrm{PnP})$ procedure per camera. We then rectify the color and infrared images and estimate the $6 \mathrm{D}$ transformation between the RGB and the infrared camera of the RGB-D sensor from a multi-view PnP procedure between the cameras.

We calibrate the extrinsic parameters of the RGB-D sensor with respect to the set of motion capture markers attached to it (see Figure 1). We attached infrared markers to the corners of the checkerboard and use the motioncapture system to detect their 3D location. The points are projected on the color image based on the currently estimated transformation between the sensor and the marker set. We minimize the error of the projection of the point markers on the color image at different locations of the checkerboard. After the calibration procedure, the point clouds recorded from the RGB-D sensor in sensor frame (Figure 1, bottom right) can be registered to the same frame as the motion-capture readings.

To acquire $6 \mathrm{D}$ pose measurements of the articulated object from the motion-capture system we attach marker sets to each of the links and place them inside the tracking volume. The motion-capture system estimates the 3D location of the infrared fiducial markers on the scene with respect to a fixed coordinate system with submillimeter accuracy and generates a $6 \mathrm{D}$ pose measurement based on the predefined model of the arrangement of the markers within each marker set. While a minimum of three infrared markers defines a marker set we use at least five markers per set to improve the accuracy of the $6 \mathrm{D}$ pose measurements and the robustness of the system against occlusions.

\subsection{Kinematic properties}

We use the $6 \mathrm{D}$ rigid-body poses to compute the ground truth of the joint parameters in an offline batch procedure. We estimate the axis of a prismatic joint by fitting a line to the timevarying positions of the child body with respect to the parent body in a least-squares sense (Figure 4, left). The computation of the kinematic state of a prismatic joint requires to define an origin. Without loss of generality we use the first pose of the child body with respect to the parent body as the origin. We calculate the prismatic joint state as the distance between the child body and this point along the fitted line.

For revolute joints, we estimate the orientation of the joint axis as a unit vector and its position as a 3D point. We obtain the plane that best fits the positions of the child body with respect to the parent body during the interaction by minimizing the squared distance of the points to the plane. The plane's normal corresponds to the orientation of the revolute axis (Figure 4, right). We then project all the points to the plane and estimate a circle using a least-squares fit with respect to the projected points. The circle's center indicates the position of the revolute axis. Without loss of generality we use the radius connecting the projection of the first pose of the child body with respect to the parent body as origin. We calculate the configuration of the revolute joint as the angle of the arc between the child body and this point along the circle defined by joint axis position and orientation.

\subsection{Interaction wrenches}

For each object we provide interactions with measurements of the interaction wrench. To obtain these measurements the humans actuate the articulated object with a tool attached to a F/T sensor (Figure 1). Because the tool with the sensor is visually salient and renders the human interaction less natural, which could negatively affect some application of our dataset, we only use it in five interactions per object. 

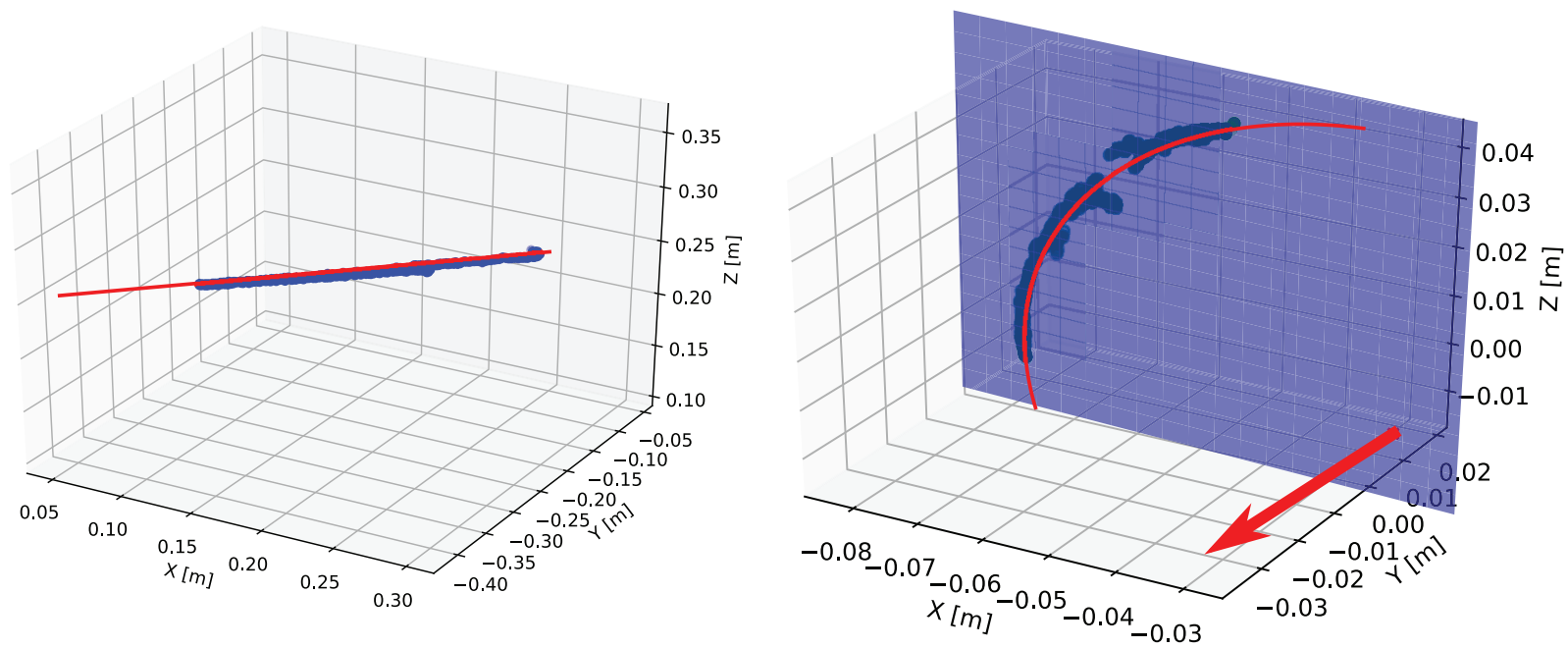

Fig. 4. Estimating the joint axis for a prismatic (left) and revolute joint (right): The blue dots show the position of the moving body, the red lines are the fitted axes. For the revolute joint (right) the orientation of the axis is obtained by fitting a plane (blue), whereas its position is based on a circle fit (red) within that plane.

The motion-capture system measures the pose of the interaction tool and we provide it as part of the interaction data. We also provide a 3D textured model of the tool with the sensor. Combining the 3D models of the tool with the sensor and the articulated object, and their measured poses, it is possible to compute the contact point of the tool and the object (assuming rigid bodies). The wrenches measured by the sensor and included in the dataset are raw values with bias in the sensor frame (Figure 1, bottom left). We measured the bias in the measurements with a calibration procedure where we align in turns one of the main axes of the $\mathrm{F} / \mathrm{T}$ sensor to the vertical direction and collect the sensor readings. The result of the calibration is the following wrench bias vector:

$$
w_{b}=\left(\begin{array}{c}
f_{b} \\
\tau_{b}
\end{array}\right)=\left(\begin{array}{c}
(-0.927 \mathrm{~N}, 1.122 \mathrm{~N}, 1.332 \mathrm{~N}) \\
(0.104 \mathrm{Nm}, 0.027 \mathrm{Nm},-0.033 \mathrm{Nm})
\end{array}\right)
$$

In order to subtract the effect of the tool from the wrench readings we measured the following dynamic properties of the elements attached to the $\mathrm{F} / \mathrm{T}$ sensor.

- Mass: $163.0 \mathrm{~g}$.

- Center of Mass: $(0 \mathrm{~cm}, 0 \mathrm{~cm},-1.5 \mathrm{~cm})$.

We also measured the transformation between the frame tracked by the motion-capture system and the measurements frame depicted in Figure 1:

$$
T_{\text {ft_meacap }}^{f t \text { meas }}=\left(\begin{array}{cccc}
0.991 & 0.052 & -0.123 & 0.019 \\
-0.060 & -0.650 & -0.757 & -0.008 \\
-0.120 & 0.758 & -0.642 & -0.001 \\
0.0 & 0.0 & 0.0 & 1.0
\end{array}\right)
$$

\subsection{Link geometries}

We use three alternative methods to generate $3 \mathrm{D}$ triangle meshes of the links of the articulated models. For small-scale objects, we use a system based on structured light (Hewlett-Packard, 2018). It projects a known light pattern onto the object to generate 3D information. The scanner acquires partial 3D models from 24 different view points using a rotating plate and integrates them into a colored triangle mesh. Before the scan we perform an initial calibration procedure to segment background from foreground. For large-scale textured objects we use the software Autodesk (2018), which reconstructs a high-definition 3D mesh by applying a multi-view geometric algorithm on overlapping color photos of the object. We take $\approx 25$ photos per object with a Casio Exilim EX-FC100 (resolution: 9 MP), located on a hemisphere centered around the object. For large-scale textureless objects such as the cabinet we generate meshes by hand using the $3 \mathrm{D}$ creation suite Blender. We post-process all models to fill holes, to remove parts of the surrounding environment, and to register them to the attached motion-capture markers.

\section{Acknowledgements}

The authors would like to thank to Johannes Wortmann, Lorenz Vaitl, and Friedrich Meckel for their help in collecting the data, and Sebastian Koch for granting us access to and helping us with the $3 \mathrm{D}$ scanner.

\section{Funding}

The author(s) disclosed receipt of the following financial support for the research, authorship, and/or publication of this article: The author(s) disclosed receipt of the following financial support for the research, authorship, and/or publication of this article: This 
work was supported by the Alexander von Humboldt Foundation and the Federal Ministry of Education and Research (BMBF), by the European Commission (EC, SOMA, grant number H2020ICT-645599), and by the German Research Foundation (DFG, Exploration Challenge, grant number BR 2248/3-1).

\section{ORCID iD}

Roberto Martín-Martín

https://orcid.org/0000-0002-9586-2759

\section{References}

Autodesk (2018) Autodesk ReMake. http://remake.autodesk.com/ about (accessed 11 July 2018).

Brown DC (1966) Decentering distortion of lenses. Photogrammetric Engineering and Remote Sensing. Available at: http:// www.close-range.com/docs/Decentering_Distortion_of_Lenses_Brown_1966_may_444-462.pdf (accessed 30 March 2019).

Calli B, Singh A, Walsman A, Srinivasa S, Abbeel P and Dollar AM (2015) The YCB object and model set: Towards common benchmarks for manipulation research. In: Proceedings of the 2015 International Conference on Advanced Robotics (ICAR). IEEE, pp. 510-517.

Cao Z, Simon T, Wei SE and Sheikh Y (2017) Realtime multiperson $2 \mathrm{D}$ pose estimation using part affinity fields. In: $C V P R$, pp. $542-550$.

COLLADA (2012) Industrial automation systems and integration - COLLADA digital asset schema specification for 3D visualization of industrial data. ISO ISO/PAS 17506:2012, International Organization for Standardization, Geneva, Switzerland.
Garcia Cifuentes C, Issac J, Wüthrich M, Schaal S and Bohg J (2017) Probabilistic articulated real-time tracking for robot manipulation. IEEE Robotics and Automation Letters 2(2): 577-584.

Hewlett-Packard (2018) SLS-3 HD. http://hp.com/go/3dscan (accessed 11 July 2018).

Kasper A, Xue Z and Dillmann R (2012) The KIT object models database: An object model database for object recognition, localization and manipulation in service robotics. The International Journal of Robotics Research 31(8): 927-934.

Martín-Martín R, Höfer S and Brock O (2016) An integrated approach to visual perception of articulated objects. In: Proceedings of the IEEE International Conference on Robotics and Automotion (ICRA), Stockholm, Sweden. IEEE, pp. 862-869.

Michel F, Krull A, Brachmann E, Yang MY, Gumhold S and Rother C (2015) Pose estimation of kinematic chain instances via object coordinate regression. In: Proceedings of the 2015 British Machine Vision Conference (BMVC), p. 181.

Motion Analysis (2018) Motion capture system. http://ftp.motion analysis.com (accessed 11 July 2018).

ROS (2018) Robot operating system (ROS), bags of messages. http://wiki.ros.org/Bags (accessed 11 July 2018).

Schmidt T, Newcombe RA and Fox D (2014) DART: Dense articulated real-time tracking. In: Robotics: Science and Systems, Berkeley, CA, pp. 342-350.

URDF (2018) Robot Operating System (ROS), Unified Robot Description Format (URDF). http://wiki.ros.org/urdf (accessed 11 July 2018). 